que depuis le rachat du Canal du Midi par l'État, le renouvellement de l'eau étant devenu plus abondant par suite de l'augmentation du trafic qui nécessite la manouvre beaucoup plus fréquente des écluses, l'intensité du développement de la flore algologique a été ralentie et celle-ci modifiée sensiblement dans sa composition par une diminution très marquée du nombre des espèces que l'on récoltait antérieurement. Tandis que les formes filamenteuses sont encore assez abondantes, un certain nombre de Chlorophycées, telles que l'Hydrodictyon utriculalum, les Pediastrum, les Desmidiées ct beaucoup d'autres formes libres et épiphytes, les Diatomées ne se montrent pas aussi abondamment qu'à l’époque où leur évolution était favorisée par l'état de stagnation du milieu, ce qui démontrerait, une fois de plus, que parmi les causes qui peuvent intervenir dans la constitution d'une flore d'Algues microscopiques, le régime des eaux et leur action mécanique exercent un effet prépondérant.

MODIFICATIONS DE LA FLORE ATLANTIQUE; ACQLISITIONS, EXTINCTIONS, PLANTES INTERHITTENTES,

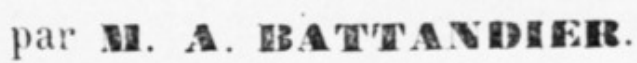

\title{
$1^{\circ}$ ACQUISITIONS ET EXTINGTIONS
}

Depuis une trentaine d'années que j'herborise en Algérie, un certain nombre de plantes montrent une tendance euvahissante, tandis que d'autres ont, pour diverses causes, disparu ou sont en train de disparaître.

Parmi les plantes envahissantes je citerai les suivantes :

Paspalum distichum L.; $P$. vaginatum Sw., Grenier et Godron, Fl. Fr. - Cette Graminée fut signalée pour la première fois en Algérie par $M$. le $\mathrm{D}^{\mathrm{r}}$ Trabut en 1885 (voir ce Bulletin). Il l'avait trouvée à l'Alma, dans un petit ruisseau. Elle était bien rare alors. Depuis, elle a envahi tous les fossés qui restent humides l'été. Elle y étouffe toutes les autres plantes et devient chaque jour plus commune.

Artemisia Verlotorum Lamotte. - Cette plante, qui est probablement une espèce asiatique introduite en Europe à une époque indéterminée, me parait bien distincte de l'A. vulgaris L. Il y a 
environ vingt-cinq ans, je trouvai à Boufarick une touffe de cette Armoise. J'herborisais beaucoup alors dans la Nitidja et je recherchais l'A. vulgaris signalé par Desfontaines. Si la plante eût été répandue, elle ne m'eût pas échappé. Aujourd'hui elle envahit tous les terrains frais de la Mitidja, orangeries, jardins, etc. Elle pullule à Blida, Boufarick, Marengo, l'Arba, etc., etc. C'est un fléau.

Conyza Naudini Bonnet. - Depuis quelques années cette plante paraît solidement établie à Aïn Taya.

Veronica Tournefortii Gmelin; V. Buxbaumii Ten. - Cette plante est depuis longtemps signalée en Algérie, mais, jusqu’à ces dernières années, je ne l'avais pas rencontrée dans la Mitidja. Aujourd'hui elle abonde à Marengo, Blida, Boufarick, Souma, etc.

Salpichroa rhomboidea Miers. - Cette Solanée de la république Argentine, échappée du Jardin d'Essai, envahit les haies des environs de cet établissement.

Atriplex halimoides Lindley et Atriplex semibaccata Rob. Br. - Ces Salsolacées, introduites avec beaucoup d'autres par M. Trabut, il y a une dizaine d'années, se sont franchemrent naturalisées à Mechéria et à Sfax.

L'A marantus albus L. est bien plus abondant qu'autrefois aux environs d'Alger, les Oxalis cernua et compressa gagnent toujours du terrain. Le Pennisetum villosum, le Lippia canescens, le Chenopodium ambrosioides se maintiennent au voisinage des habitations. La première de ces plantes, cultivée avec d'autres Pennisetum, comme piège à altises, va probablement se répandre beaucoup.

L'Eucalyptus rostrata, se ressemant de lui-même tend à devenir subspontanẻ; il en est de même de l'Acacia horrida, du Nicotiana glauca, etc.

Le Franzeria artemisioides Willd. est apparu cette année à El Biar.

A côté de ces acquisitions, bien des stations ont disparu, tant par suite du développement considérable de la ville d'Alger, que de l'extension des cultures intensives, en particulier de la vigne.

Le Statice virgata L., qui couvrait une dizaine de mètres de falaises à Bab-el-Oued, a été détruit par l'établissement du boulevard Front de mer. Les constructions ont de même fait disparaître 
le Lemna gibba à Mustapha, l'Allium trifolialum Kunth au Ilamma. Le Sinapis procumbens Poiret, qui avait une vaste station a I'Agha, n'y occupe plus que quelques terrains à bâtir. Le Trifolium clypeatum L. et le Malva oxyloba Boissier, plantes adventices demeurées longtemps à Mustapha, ont totalement disparu. Le Cerinthe minor L., qui avait été signalé en Algérie par Desfontaines, a été très réellement cueilli au Ruisseau, près d'Alger, par Clauson et par Duval-Jouve. Cette plante, probablement adventice aussi, n'y existe plus. L'existence du Dianthus tripunctatus Sibth. et Sm. se trouve bien menacée dans son unique localité algérienne de Saint-Eugène. L'Onopordon algeriense Pomel, connu seulement à la Pointe Pescade, le.sera à son tour bientòt.

Les plantations de vignes ont fait disparaitre le Cistus crispus de Saoula, le Themeda Forskhalii de la Reghaïa. Les marais de la Rassauta et du Fort de l'Eau, qui contenaient tant de plantes spéciales des plus intéressantes, sont de plus en plus resserrés par les cultures maraichères, et l'accès en devient difficile. Il en est de mème des riches stations de Guyotville, presque toutes remplacées par des vignes ou des cultures de primeurs. L'Hesperis ramosissima Desf., le Peristylus cordatus Lindley, y sont devenus presque introuvables

Les cultures modifient beaucoup la flore primitive. Les broussailles disparaissent et avec elles beaucoup de plantes qui ne subsistaient qu'à leur abri. La plupart des plantes vivaces ou bisannuelles sont détruites; beaucoup de plantes annuelles sont a contraire favorisées.

Beaucoup de plantes à bulbes : Scilla divers, Merendera, Colchicum, etc., qui formaient de véritable tapis à l'automne, ne résistent pas aux labours. Il peut arriver que des espèces très voisines se comportent très différemment vis-à-vis des cultures. C'est ainsi que le Convolvulus arvensis L. est à peu près indestructible, tandis qu'un seul labour détruit à jamais le Convolvulus Durandoi Pomel. Le Gladiolus byzantinus Miller, qui existe presque seul dans les bruussailles, devient rare dans les cultures oú, pullule au contraire le $G l$. segetum L. 


\section{PLANTES INTERMITTENTES}

Le Damasonium Bourgcei Cosson, très abondant certaines années dans la Mitidja, devient ensuite introuvable pendant de longues périodes. Cette année, la plus pluvieuse que l’on ait vue depuis longtemps en Algérie, il est apparu abondamment à Aïn Taya, touchant ma propriété, dans une localité où je ne l'avais pas vu depuis dix ans, bien que j'y aille à peu près toutes les semaines. J'ai pu y commencer l'étude des conditions nécessaires à son développement.

Ce Damasonium, et il en est probablement de mème des D. stellatum et polyspermum, ne germe que dans les caux stagnantes peu profondes et qui persistent longtemps sans grand changement de niveau. Je ne l'ai pas vu germer à plus de vingt centimètres de profondeur. Il manque toujours dans les fossés un peu profonds où pousse l'Alisma Plantago. Pendant ces dix dernières années j’ai vu plusieurs fois se reformer cet étang temporaire, mais le Damasonium ne s'y était point développé, l'eau n'ayant pas séjourné assez longtemps. Tant que la plante est inondée, elle ne forme que quelques feuilles flottantes clliptiques atténuées aux deux bouts et longuement pétiolées. Il faut que l'eau disparaisse pour que la rosette de feuilles radicales cordées-ovales et les hampes florifères se développent. Les feuilles nageantes disparaissent alors très vite. On conçoit que tout cet ensemble de conditions : eaux stagnantes, peu profondes, persistant longtemps, disparaissant en temps voulu, soit assez rarement réalisé.

Avec le Damasonium s'étaient développés, dans la même station, l'A lisma ranunculoides L. et le Peplis nummularicefolia Lois., que l'on y trouve rarement aussi. Le Laurentia Micheli DC. s'y était multiplié d'une manière inusitée.

Le Ranunculus batrachioides var. pusillus parait exiger des conditions semblables à celles du Damasonium Bourgcei, mais avec un tirant d'eau bien moindre, la plante étant très petite. Aussi ne la trouve-t-on que sur les montagnes où les conditions climatériques permettent à d'aussi faibles épaisseurs d'eau de persister un temps suffisant. Cette Renoncule possède de petites feuilles flottantes identiques de forme à celles du Damasonium Bourgcei. Ces feuilles n’ont pas été figurées dans les Illustrationes 
Florce atlantica, pl. 1. Mon regretté correspondant Julien, qui avait découvert une station de cette plante au djebel Ouach, ne put l'y retrouver les années suivantes.

Beaucoup de plantes des mares, surtout dés mares temporaires, semblent exiger pour leur développement des conditions particulières qu'il serait intéressant d'étudier.

L'Heliolropium supinum occupe le fond de petites mares éphémères, le Polygonum viviparum ne se trouve que dans celles qui ne tarissent jamais. L' Illecebrum verticillatum et les Peplis veulent des mares oú l'eau persiste longtemps, etc.

Le Rumex algeriensis Barratte et Murbeck ne se trouve que dans des mares herbeuses, ou l'eau persiste jusqu'en juillet, sans être trop profonde ni courante. Le $R$. crispus L., espèce voisine, vient au contraire partout où le sol est suffisamment humide.

Un petit nombre de mares nourrissent, en peuplements compacts, le Chrysanthemum Clausonis Pomel, Kremeria paludosa Durieu, alors qu'il manque dans toutes les autres.

Les plantes des mares temporaires ne sont pas les seules qui présentent ainsi des intermittences. Dans les pays, comme le Sahara, à pluies très irrégulières, presque toutes les plantes annuelles en présentent; mais il s'en produit un peu partout. Dans un ravin de Mustapha, voisin de mon habitation, le Coronilla atlantica Boissier et Reuter ne se développe complètement que les années où le printemps est Irès pluvieux, les autres années la plante sèche avant de fleurir. Je ne connais aucun moyen de transport qui puisse amener dans ce ravin les fruits de celte Hédysarée, il faut donc qu'ils ne germent pas tous la même année. C'est d'ailleurs un fait général chez les plantes sauvages, fait des plus utiles pour la conservation de l'espèce. Des semis expérimentaux m'ont démontré que beaucoup de semences de plantes sauvages germent dans des espaces de temps très irréguliers soit dans la mème année, soit dans des années différentes. En Algérie, une averse trop précoce amène souvent d'abondantes germinations qu'un retour de sécheresse détruit entièrement; quand les pluies viennent ensuite, de nouvelles germinations tout aussi abondantes se produisent. Tous les agriculteurs savent que, pour détruire les herbes d'un champ, il faut les empècher de grainer plusieurs années de suite. Pour le Damasonium Bourgaci, il faut évidemment 
que ses graines conservent longtemps leurs propriétés germinatives.

Certaines années, j'ai vu près d'Alger le Trifolium suffocatum L., rare d'ordinaire, devenir fort abondant.

\section{NOTES D'HERBORISATIONS, par M. A. BATTANDIER.}

Delphinium silvaticum Pomel. - Cette plante, qui n’élait connue que dans l'Est de l'Algérie et la Tunisie, a été trouvée par le $\mathrm{D}^{2}$ Trabut à la Reghaïa, près de la ferme de M'sera.

Ranunculus batrachioides var. pusillus; R. pusillus Pomel, Nouv. mat.; R. xantholeucos Cosson et Durieu, forma pusilla, Illustr.Fl. atl., tab. I. - J'ai étudié cette année cette plante dans sa station classique de Terni, près Tlemcen. Son nanisme tient à la station où elle pousse. Toutes les plantes y sont également naines. Le Juncus bufonius n'y dépasse pas 4 centimètres. En cherchant aux environs, j’ai trouvé une station où la plante atteignait 15 à 20 centimètres, taille supérieure à celle du Ranunculus de Tiaret, considéré comme le type de l'espèce. Cependant les deux plantes restent différentes. Celle de Terni a un port plus élancé, ses pétales sont plus étroits. Il n'y a pas, à mon avis, de quoi rétablir les deux espèces de M. Pomel, mais bien deux variétés. La plante de Terni a été retrouvée par M. Julien au djebel Ouach, à Constantine.

Ranunculus lateriflorus DC. - Forèt d'Afir, et en général mares des montagnes.

Papaver dubium L. var. albiflorum - Variété remarquable par ses très petites fleurs blanches, lavées de mauve, sans tache à la base. Moulin à huile du Tleta, près du pont de la Tafna, assez abondant. J'avais cru d'abord retrouver le $P$. malveflorum Doumergue (Association française 1896, congrès de Carthage), mais sa description ne concorde pas avec cette plante.

Ionopsidium heterospermum Batt., Bull. Soc. bot., 1896, p. 256. - Pentes boisées du djebel Tenouchfi, Mazer. - Mai. Cette plante, assez abondante, est moins puissante que ne me l'avait fait croire l'échantillon géant rapporté par M. le Dr Trabut, à l'époque où je la décrivis. Elle a, dans les sous-bois, l'aspect du 


\section{$2 \mathrm{BHL}$ Biodiversity Heritage Library}

Battandier, Jules Aimé. 1904. "Modifications De La Flore Atlantique; Acquisitions, Extinctions, Plantes Intermittentes." Bulletin de la Société botanique de France 51, 345-350. https://doi.org/10.1080/00378941.1904.10829140.

View This Item Online: https://www.biodiversitylibrary.org/item/8673

DOI: https://doi.org/10.1080/00378941.1904.10829140

Permalink: https://www.biodiversitylibrary.org/partpdf/160335

\section{Holding Institution}

Missouri Botanical Garden, Peter H. Raven Library

\section{Sponsored by}

Missouri Botanical Garden

\section{Copyright \& Reuse}

Copyright Status: Public domain. The BHL considers that this work is no longer under copyright protection.

This document was created from content at the Biodiversity Heritage Library, the world's largest open access digital library for biodiversity literature and archives. Visit BHL at https://www.biodiversitylibrary.org. 